\title{
SURVEY OF COMMERCIAL MANUAL CONTROLLERS FOR A GENERIC TELEROBOTICS ARCHITECTURE
}

\author{
Thomas E. Deeter and Dean L. Schneider \\ Air Force Institute of Technology \\ Department of Electrical and Computer Engineering \\ Wright-Patterson AFB, OH 45433-7765
}

\begin{abstract}
The purpose of this study was to determine an input device for the Air Force's generic telerobotics architecture for large aircraft maintenance and repair. Our area of concern was the human to machine interface, more specifically, which manual controller or input device should be used for the specified tasks in this architecture. We mailed a survey to 68 companies in order to compile a list of possible input devices that the telerobotics architecture could use. Thirty two companies responded giving us enough data to generate a list that described the physical traits of the input devices. The physical traits were divided into two main categories. The first category, called device category, deals with items such as external power, type of communication interface, and the number of degrees of freedom. The second category, called user category, discusses items such as cost, size, weight, and dexterity. We then divided the required tasks that the Air Force's generic telerobotics architecture must perform into actions and analyzed them to generate a list of traits required by an input device. Both the task analysis and device listings were combined mathematically to form a performance table which compared the input devices that could perform each individual action.
\end{abstract}

\section{INTRODUCTION}

Since the Air Force is currently down-sizing and cutting back its infrastructure, the development of a manual controller for a specific situation is expensive and may not be the best option. Another option is to look for a less expensive commercial manual controller that is already available. The proposed methodology must locate possible manual controllers which are available in the commercial market. This research has surveyed companies that could possibly manufacture manual controllers which could be used in a general telerobotics architecture. The replies from the survey was tabulated. The tables were not included in this paper due to length restrictions. The tables can be transferred from the AFIT archives by anonymous fitp. Contact the authors for more details.

\section{DEVICE AND TASK ANALYSIS}

This section will begin with a discussion of the survey responses and how the information from the responses was tabulated. It will also discuss the tasks involved in the generic architecture.

\section{A. Commercial Survey of Companies and Device Analysis}

This section will present the survey information obtained from many different companies. The information was tabulated to allow easier comparison of each device to a particular task. In most cases, the tabulated information was multiplied and summed with the task analysis information. In some cases, the particular item was an elimination item. Once unqualified devices were eliminated, the task analysis table was then multiplied with the device analysis tables in matrix fashion. The resultant table contains the results of the multiplication and the device with the lowest number will be considered the best device. The resultant matrix will contain device results for each task; thus, a device that is the best for one task may not be the best for another task

1) Survey Results: Early in this research, letters requesting any information on any possible input devices were sent to over 60 companies. The letter requested that the company send information on any devices that could possibly be used in a telerobotics applications or in any human to machine interface system. Several companies responded by letter and/or brochure. The information from the responses was tabulated to give a grading of the input devices.

Tha infermation was divided into two main categories or point of views. The first category is how a device designer would judge the devices. This category would look at issues or items such as electrical interface, communications type, number of degrees-of-freedom (DOF), resolution of each DOF, etc.. The second category is concerned with how a user would judge the device. The user is defined as the person using the device. The issues or items observed in this category are size of the device, dexterity, type of grip etc. Each category will be explained in detail.

2) Engineer Category. The engineer category is concerned with issues that an engineer would maximize to make the optimal device. The table located in the AFIT 
archives contains the tabulated information. We tabulated the information into six items.

The first item in the table is power. The less the power the better the device. A one in the power item of a device represents no external power supply required while a ten represents a large power supply such as $1 \mathrm{~mA} @ \pm 15$ volts A five represents a five volt external power supply required to operate the input device.

The second item is communication interface type. This item is an elimination cell which will either allow the device to be tested or will eliminate the device from the test. The only possible types of communication interface for this survey are serial, parallel, analog, switches and/or other. Some devices may have two or more different types of communication interfaces, while others may have none which would require additional hardware to interface to the system. An analog output device will require additional circuitry to interface to a computer, such as an A/D board or an analog to serial interface card. This situation may be misleading to the outcome of "optimal device" because the cost to interface a device to the computer will be included in some devices but not others. Thus, a cost adjustment must be made to even the score. Based on an average of interfaces and A/D chips, the price should be increased by $\$ 100$ and the weight increased by one pound. These increases are only estimates and are not the cost and weight of a constructed product. The switch interface type is used to indicate a toggle switch joystick. A toggle switch joystick is a joystick that operates only by opening and closing switches. There are only on or off signals from a toggle switch joystick.

The next item in the table is DOFs which is the number of DOFs the device can control. This item does not include the number of buttons, even though buttons can be used to generate a DOF.

The resolution per DOF item is defined as follows. Some devices use open-close switches to develop a DOF signal (toggle switch joystick); thus, the resolution for that DOF is one bit which corresponds to a ten for this item. Other devices convert sensor output to a hex value that can be read by a computer. See Table 1 to determine the resolution per DOF.

Since the computer must read the input device signal and cannot read an analog signal directly, the value of infinity was included only to grade the device. In some cases, the actual resolution would be determined by the type and resolution of the interface device. For example, if a device supplies a voltage then it could have an infinite resolution.

The device would then be interfaced to the systems computer. For this example, assume an $\mathrm{A} / \mathrm{D}$ is used to interface the device to the computer. The actual resolution
Table 1 DOF Resolution

\begin{tabular}{|c|c|}
\hline Range of hex Values & $\begin{array}{c}\text { Resolution per } \\
\text { DOF }\end{array}$ \\
\hline 1 & 10 \\
\hline $2-16$ & 9 \\
\hline $17-64$ & 8 \\
\hline $65-144$ & 7 \\
\hline $145-256$ & 6 \\
\hline $257-400$ & 5 \\
\hline $401-576$ & 4 \\
\hline $577-1024$ & 3 \\
\hline 1025 or Greater & 2 \\
\hline Infinity & 1 \\
\hline
\end{tabular}

would be the range of hex values the A/D can supply to the computer. Some devices only output a one if a certain distance is traveled. This type of device, mouse for instance. is normally rated in dots-per-inch (dpi) or pulses per revolution (ppr).

The next item in the engineer table is the number of buttons. A button on a device can be very useful. They could be used as an emergency shutdown, mode change, or a simple check to ensure the user has a grasp on the grip. Buttons could also be used to generate an additional DOF.

The last item on the engineer table is the type of device. The possible values of this item are joystick, mouse, trackball, or other. Other describes an input device that does not fit the other three device types.

Three items that were not included in the engineer table were communication speed, signal stability, and temperature tolerance. The items were not included for two reasons. First. there was little information available for these items. Secondly, if the information was available, it was well within the human limits of operating an input device.

3) User Category: The other category that the device information is divided into is user analysis. The user analysis is concerned with how the user judges the device. An ideal device will feel transparent to the user while a bad device will cause the user a tremendous amount of grief. Though most items in this category are subjective, they are most likely more important than the items in the engineer category. In most cases, the items in the engineer category can be adjusted (engineering trade-off) to the requirements of the system. If the device does not feel right to the user, then the task will not be performed in the best manner.

The first item in this category is the cost of the device. The values in this item will go from one to ten where one is the lowest price and ten is the highest price. Since the range of prices is large, this item will be divided into 20 parts instead of ten like the other items. The divisions are shown in Table 2 . 
Table 2 Device Cost Rating

\begin{tabular}{|c|c|}
\hline Price $(\$)$ & Item Number \\
\hline $0-100.00$ & 1 \\
\hline $100.00-200.00$ & 1.5 \\
\hline $200.00-300.00$ & 2 \\
\hline $300.00-400.00$ & 2.5 \\
\hline $400.00-500.00$ & 3 \\
\hline $500.00-600.00$ & 3.5 \\
\hline $600.00-700.00$ & 4 \\
\hline $700.00-800.00$ & 4.5 \\
\hline $800.00-900.00$ & 5 \\
\hline $900.00-1000.00$ & 5.5 \\
\hline $1000.00-2000.00$ & 6 \\
\hline $2000.00-3000.00$ & 6.5 \\
\hline $3000.00-4000.00$ & 7 \\
\hline $4000.00-5000.00$ & 7.5 \\
\hline $5000.00-6000.00$ & 8 \\
\hline $6000.00-7000.00$ & 8.5 \\
\hline $7000.00-8000.00$ & 9 \\
\hline $8000.00-9000.00$ & 9.0 \\
\hline $9000.00-10000.00$ & 9.5 \\
\hline $10,000.00$ or greater & 10 \\
\hline
\end{tabular}

The next item in the user table is reliability. This item is difficult to obtain from a company catalogue or brochure. There are certain characteristics that will help in determining reliability. A device is only as good as its weakest part. For instance, if the joystick sensors are potentiometers, then the reliability would not fare as well as a joystick with inductive sensors, on average. In some cases, reliability information can be obtained on the device from a catalogue or brochure. Such information might be described as cycle limit. Other brochures may describe the reliability in terms of Mean-Time-Between-Failure (MTBF). For the purpose of this research, the reliability will be a number between one and ten. A one represents good reliability and a ten represents poor reliability.

The third item in the User table is dexterity. Dexterity describes the physical readiness and grace of a device which can be very subjective. A dexterity measure can be determined by observing the physical characteristics of a device. The size of the tool is a good measure. If the tool size is small, then the dexterity measure is small. Likewise, if the tool is large, then the dexterity measure is large. The tool is the object that is in direct contact with the human while the device is being used. The amount of travel the tool has is another good measure. In the case of a joystick, if the tool travels \pm 20 degrees then the dexterity measure would not be as good for a tool that travels \pm 60 degrees. In the case of a trackball style device, the amount of travel is infinite. These two physical characteristics should be combined in a way that will not cause one characteristic to out weigh the other. The following guidelines are given to aid in determining the dexterity measure. The size of the tool and the travel of the tool is broken into five sizes a: shown in Table 3 .

Table 3 Tool Length and Travel Measurements

\begin{tabular}{|c|c|c|}
\hline Tool Length & $\begin{array}{c}\text { Measure- } \\
\text { ment }\end{array}$ & $\begin{array}{c}\text { Tool Travel } \\
\text { (Degrees) }\end{array}$ \\
\hline under $1 \mathrm{~cm}$ & 5 & under 20 \\
\hline 1 to $4 \mathrm{~cm}$ & 4 & 20 to 60 \\
\hline 4 to $8 \mathrm{~cm}$ & 3 & 60 to 100 \\
\hline 8 to $12 \mathrm{~cm}$ & 2 & 100 to 180 \\
\hline longer then $12 \mathrm{~cm}$ & 1 & over 180 \\
\hline
\end{tabular}

The two measures should be added to make the total dexterity measure. If a device has a tool that is five $\mathrm{cm}$ Iong, a measure of three. and travels +35 degrees, a measure of three, then the total dexterity measure would be six. A trackball tool would have a tool length of zero and a tool travel of infinite thus the total dexterity for a trackball is six. The same rules that pertain to the trackball can be used for the mouse. The mouse tool length is zero, a measure of five and the mouse travel is infinite which corresponds to a measure of one. The total measure for a mouse is six. The dexterity measure for a trackball and a mouse are the same which, depending on application and/or personal preference, may not be true. There are arguments for each device why it should be better than the other. For this research, both devices are considered to have the same dexterity measure.

The next item in the user table is the grip. This elimination item will identify what kind of grip the device has and the task table will allow those desired grips to be tested.

The next two items in the user table are size and weight. For most telerobotics tasks, size and weight are not an issue; however, if the device must be carried into space or placed in a small room, then size and weight become an issue. For this research, the size and weight was distributed linearly into ten equal parts. The smallest and lightest device being a one and the largest and heaviest being a ten. To determine the size, the entire workspace was considered. For instance, a joystick has a base and a tool. The size of a joystick is the total height of the joystick, height of the tool plus the height of the base, times the square of the maximum of either the tool travel width distance or the base width.

The next item, which is an elimination item, is selfcentering. The self-centering item is a yes or no item that is used to determine if additional safety precautions must be used to interface the device. The basic question this item answers is will the device fall or move if the user removes the hand or lets go of the device. This item also tells if the device is a position input or a velocity input. A selfcentering device is usually thought of as a velocity control device and a non self-centering device is a displacement or position input device. If the device centers itself or returns to a standard position, the item is yes. 


\section{B. Task Analysis}

This section will discuss the tasks that must be performed by the Air Force's generic telerobotics project. It will discuss the process level tasks by breaking them into specific actions. The specific actions will be analyzed to develop a set of desired input device requirements. The desired requirements will then be described in a manner that matches the device tables.

The tasks for the Air Force's generic telerobotic architecture are defined in a report written by the Jet Propulsion Laboratory (JPL)[1]. The document lists si. process level tasks that must be accomplished by the system and must use an input device.

1) Task Requirements: The first process level task listed in the JPL document is painting of the $\mathrm{C}-5 \mathrm{~A} / \mathrm{B}$ exterior in a dedicated hanger facility. The requirements that apply to the input device for this task are as follows:

1. Perform $90 \%$ of paint application with little or no repainting.

2. Apply primer between $0.5-1.5$ mils \pm 0.5 mils.

3. Apply paint between $2.0-3.0$ mils \pm 0.5 mils.

4. Perform flare offs and other painting patterns to smoothly merge adjacent painting areas regardless of drying.

5. Provide separation/standoff distance accuracy of \pm 1.0 inch, repeatability of $98 \%$ in supervised-autonomous control modes.

6. Provide end-effector tool angle of incidence normal to the surface being painted, with an accuracy of \pm 5 degrees in shared, supervised-autonomous and autonomous control modes.

The second task is painting of removed parts in a robotic workcell. The requirements for this task are the same as the previous task with the exception of the flare off requirements. It is assumed that the entire part will be painted thus no flare offs are required. Another difference from the previous task is the mobile/crane is not required to place the manipulator into place. It is assumed the part is in place and the manipulator has full access to that part.

The third task is paint stripping of a $\mathrm{C}-5 \mathrm{~A} / \mathrm{B}$ in a dedicated hanger. The specific requirements for this task are as follows:

1. Maintain a standoff distance of 18 to 24 inches with a \pm 2 inch tolerance and accuracy of \pm 1 inch with a repeatability of $98 \%$.

2. Direct and control particulate (e.g. plastic material bead, water, glass bead, $\mathrm{CO} 2$ ) blast pressure and material flow rate.

3. Provide selectable end-of-arm tooling angle of incidence to the aircraft surface normal of $0-45$ degrees, with an accuracy of \pm 5 degrees in shared, supervised autonomous and autonomous control modes.
The fourth task is surface finishing in the form of removing material from patches and polishing metal to a high gloss finish in a robotic workcell. This task is unique among the other tasks because surface contact is required. The other tasks require a standoff distance. The specific tasks are as follows:

1. Provide surface contour following for unmodelled parts with a selectable force application range in shared and supervised-autonomous control modes.

2. Accommodate the removal of 10-20 mils (not exceeding the paint alodine boundary) of material, with and application force boundary no greater than that required to remove the paint.

The fifth task is surface cleaning of removed parts in a robotic workcell through application of a bicarbonate of soda particulate stream. The specific requirements for this task are as follows:

1. Provide separation distance accuracy of \pm 1 inch repeatability of $98 \%$ in shared and supervised-autonomous control modes.

2. Provide selectable end-of arm tooling angle of incidence to the part surface normal of 0-45 degrees, with an accuracy of \pm 5 degrees in shared and supervised-autonomous control modes.

2) Description of Actions: To aid in developing a set of specific requirements for an input device, a set of actions are defined to accomplish a group of tasks. The actions required for the tasks and an explanation of the requirements are listed below.

1. Grasp or attach the proper tool to the manipulator. Since all objects in this action can be structured, the input required by the human is a simple get tool command. An easy way to implement such a command would be to have a list of icons or a menu that the human can choose. For example, a menu with such options as attach paint gun, attach grinder, attach water sprayer, attach bicarbonate stripper, and attach polish tool could be used so the user could drag the pointer to the appropriate item on the menu and the selected tool would be grasped or attached to the manipulator. Since human to screen interface is required, at least a two DOF input device is needed. The device must also have one or more buttons to activate the icon and/or menu item. There are no limiting factors on power, dexterity, grip, size, weight, self-centering, resolution, and type of device other then user preference. The limiting factors will be comm. type, cost, reliability, DOFs, and number of buttons. To interface the device to AFIT's system, the comm. type must be serial, parallel, or A/D which allows most devices to be used. The cost should always be a minimum and the reliability should always be a maximum. The task analysis table shows the resulting elimination cells and weights for the desired input device. 
Table 4 Task Analysis

\begin{tabular}{|c|c|c|c|c|c|c|}
\hline Action & 1 & 2 & 3 & 5 & $6 \mathrm{a}$ & $6 \mathrm{~b}$ \\
\hline Power & 5 & 5 & 5 & 5 & 5 & 5 \\
\hline Comm. Interface & $\begin{array}{c}\text { Seria } \\
1\end{array}$ & Serial & Scrial & Serial & Strin! & Scrin! \\
\hline Cost & 100 & 100 & 100 & 100 & 10 & 10 \\
\hline Reliability & 10 & 10 & 10 & 10 & 10 & 10 \\
\hline Dexterity & 1 & 50 & 50 & 1 & 100 & 100 \\
\hline Grip & $\mathrm{X}$ & $\mathrm{X}$ & $\mathrm{X}$ & $\mathrm{X}$ & $\mathrm{X}$ & $\mathrm{X}$ \\
\hline Size & 1 & 1 & 1 & 1 & 1 & 1 \\
\hline Weight & 1 & 1 & 1 & 1 & 1 & 1 \\
\hline Self Centering & $\mathrm{X}$ & $\mathrm{X}$ & $\mathrm{X}$ & $\mathrm{X}$ & $\mathrm{Y}$ & $\mathrm{Y}$ \\
\hline DOF & $\geq 1$ & $\geq 3$ & $\geq 3$ & $\geq 1$ & $\geq 3$ & $\geq 2$ \\
\hline $\begin{array}{c}\text { Resolution per } \\
\text { DOF }\end{array}$ & $\mathrm{X}$ & $\leq 6$ & $\leq 6$ & $\mathrm{X}$ & $\leq 6$ & $\leq 6$ \\
\hline \# Buttons & $\geq 1$ & $\geq 0$ & $\geq 1$ & $\geq 1$ & $\geq 1$ & $\geq 1$ \\
\hline
\end{tabular}

or in straight lines. The trajectories required may be difficult to compute and accomplish in some areas of the aircraft.

To pick the corners of the workspace, the user must move the robot to those corners. The user will most likely be looking at a TV monitor and moving the manipulator to the desiredl point. If a force feedback device is not used, then an indication of contact or proximity tolerance must be supplied to the user. The input device must be at least a three DOF device. The user could move the manipulator into location in a two dimensional space then activate some kind of force control or proximity algorithm that would move the manipulator until it contacted the aircraft. In either case, the user must maneuver the manipulator into a point. The point may be an adjacent point to

2. Gross movement of manipulator to the task area. The manipulator will be mounted on some sort of movable base. The options are a tele-crane, gantry, or mobile system [1]. In any case the manipulator, once loaded with the appropriate tool, must be moved to the aircraft and positioned such that the work can be accomplished. Since this is a rough movement requirement, the accuracy and repeatability will not be a factor when deriving the user input specifications. The problem with this action is the requirement that $90 \%$ of the aircraft must be accessible by the manipulator and the aircraft is not easily accessed in all locations. The important items are listed in the task analysis table (Table 4) which shows the elimination items and weights for the corresponding device analysis tables. The limiting factors for this action are comm. type, cost, reliability, dexterity, and DOFs. The dexterity is more important on this task because of the mobility problem. To handle the any position, a three DOF or higher input device is required.

3. System instructions from operator through interface to system. This specific action will be used if supervisorautonomous modes of operation is desired. The user will input a set of coordinates, some additional instructions, and the manipulator will perform the task. An example of this can be explained by describing this action while a user is stripping an aircraft using bicarbonate. The user would pick four or more corners on a video screen that represents at least four corners of the area that needs to be stripped. The computer would then compute the best possible trajectory. Keep in mind the trajectory is only two dimensional. The third dimension is controlled by either a force sensor or proximity sensor using a concept called shared control. Shared control has been implemented at AFIT to determine its applicability to this scenario. Again, the problem with this action is the requirement of completing $90 \%$ of the aircraft. The aircraft is not square the last section of work area or a point that is close to an obstruction on the aircraft. Thus accuracy must be maintained to prevent aircraft damage. The end effectors used in this group of tasks inherently have some tolerance. For example, the bicarbonate stripper has a spray width of approximately three to four inches. The paint spray has a width of one to four inches depending on the type of sprayer used. Even with these tolerances, the points selected by the user should have an accuracy of 0.25 to 0.1 inches. This accuracy can be easily obtained with any device that is a displacement device. The software could be modified to control the manipulator distance for an associated distance from the input device. An example of this would be to use a mouse to move the manipulator one millimeter. The software could require the mouse be moved several inches before the manipulator is moved a single millimeter. On the other hand, a single pulse from the mouse could represent several inches to the manipulator. If the above could be adjusted by the user, then perhaps the user could select a more accurate movement when needed and a faster movement when accuracy was not an issue. Thus, some sort of throttle control could be desirable to the user. An accepted method of adjusting the speed of the mouse is called ballistic speed adjustment. This means the more the mouse is moved in one direction the faster the pointer moves.

4. Monitor task using sensor devices and appropriate feedback to user. This action does not require an input device to accomplish. It will be handled by the system and appropriate output devices.

5. Error detection and recovery. This action requires one input from the user. A safety button or switch should be used to accomplish this action. A button on the input device would be a viable option to accomplish this action, yet risky. If the user accidentally pushed the wrong button 
on the input device, then an unwanted shutdown would occur. It is recommended that a separate, rather large, button be used to accomplish this action. The large button would be easy to locate and activate under an emergency or a fault situation.

6. If required, fine motion of manipulator to accomplish required task. This action is required if for some reason the equipment fails, the system cannot handle one of the many variables, or the user deems it necessary to control the manipulator manually. Since the orientation and position is a required control variable (see task requirements above), a six DOF input device or two. three DOF input devices are needed with all possible sensor data displayed to the user. Such a system would give complete control to the user. However, in some cases the input device would only control two DOFs while the proximity or force sensor controls the others. Since this action can be divided into two different methods of accomplishment. it will be separated into two actions. The first action (6a) is complete control of the manipulator by the user. The second action ( $6 \mathrm{~b}$ ) will be shared control between the input sensors and the computer, and the user. As stated earlier, action 6 a requires a six DOF input device that is fairly dexterous and easy to use by the user. Action $6 \mathrm{~b}$ will only require a two DOF input device. Action $6 b$ has been implemented in the AFIT robotics lab to demonstrate shared control while simulating painting an aircraft skin. This action requires a rather dexterous two DOF input device and at least one button to activate the sprayer or stripper. The user can control the paint gun in two dimensions while the computer and input sensor(s) (proximity or force sensor) control the other DOFs.

The task analysis table (Table 4) displays the appropriate weights and eliminations for each action. Some items described in the device tables are not included in the task analysis table because they were not a concern for the list of actions required to accomplish the tasks. Some items are the same for all actions which is due to the action requirement and/or the hardware.

Some items are called elimination cells because these items describe a hard limit that if not met then the derice must not be included in the table. The numbers in the task analysis items indicate a weight associated with that item.

The rows in the task analysis table (Table 4) represent the weights for a corresponding item in the device tables The first row is power. Since our system is maintained on the ground in a controlled environment, the weight for this item is five for all actions. The second row is comm. interface. This elimination item requires the device to have a serial output of some sort. As mentioned earlier, an analog device can be connected with the proper hardware which will add to the cost, weight, and size of the device. The third row is cost. Cost is important to the Air Force so a weight of 100 is in most of the actions. However, the cost is lowered for actions that require high dexterity to make the dexterity weight more valuable. The fourth row is the reliability. The weights for all actions for this item are tens. A ten indicates this item is an important factor, but not the most important. The fifth row is grip. The grip does not matter in this analysis as long as the actions are able to be completed reasonably and is so indicated by an $\mathrm{X}$ in all actions in the fifth row. The sixth row is dexterity. Dexterity is not an issue for the first five actions; thus, the weight is only ten. Howcrer. for the last two actions in the sixth row of the task analysis table. dexterity is important which is shown by the weights of 100 . The seventh and eighth rows are size and weight. respectfully. Since the input device will be in a controlled environment with unlimited room and unlimited weight constraints, the weights for this item are ones. The ninth row is self-centering. Since position control or velocity control is not an issue, the elimination item is labeled as don't cares for all but the last two actions. The last two actions contain a yes because if the user were to let go of the input device, then the aircraft may be damaged. The last three rows of the task analysis table represent minimum or maximum requirements. These last three rows are used as elimination items to pick only the input devices that meet or exceed the requirements.

\section{COMBINING THE DEVICE AND TASK TABLES}

This section will combine the device tables and the task table and generate a resultant table that represents the input devices to task relationships (in AFIT archives). The end result is a set of possible input devices per action. The mathematical procedure is discussed and issues that must be resolved before the tables can be combined. A simple example of comparing one device to a task is also accomplished to aid the users understanding of the mathematical procedure.

\section{A. Combining Issues}

Before the tables can be combined, some issues must be resolved. The first issue is how the analog input devices will be interfaced to the system. One possible solution is to use an $A / D$ to digitize the analog signal. $A$ fast reliable $A / D$ board will be relatively expensive compared to some of the input devices evaluated in this report. However, if expense is not a large factor, then the A/D board is a viable option. The A/D board would most likely be the fastest and most reliable of the options. Another option is to construct an interface device. The interface device interfaces the input device to any possible input port on a computer. Some possible input ports are serial port (RS-232, RS-422), bus port, parallel port, etc. A common interface is from the input device to a serial port; probably because most computers have a serial port. Like the A/D board, a fast and reliable interface would be relatively expensive and would not be as fast as the A/D board. The reason the interface takes longer time than the $\mathrm{A} / \mathrm{D}$ board is because the interface device must digitize the signal then supply the serial line with a stream of bits. Where as the A/D board just digitizes 
it and supplies the data to the data bus of the computer. The actual time, or absolute time, of either interface is not the issue. The issue is the speed of one verses the other or relative time. For the purpose of this research, if an input device uses the $A / D$ board then the price will be increased by $\$ 100.00$, the comm. speed will be increased by 0.0005 seconds, and the DOF resolution will have a maximum resolution of 255 which corresponds to a six in the device tables. If the serial interface device is used, then the cost will be increased by $\$ 100.00$, the comm. speed will be increased by 0.0008 seconds, and the maximum resolution will be six. These numbers are only estimates.

\section{B. Mathematical Procedure}

The combining of the device tables and the task table is accomplished by a mathematical approach. The device tables are summed by concatenating the rows making a $\mathrm{m}$ by $n$ matrix where $m$ is the number of items and $n$ is the number of input devices. The complete device table (matrix) is then multiplied by the task analysis table (Table 4) which acts as a weighting matrix. The resultant matrix is the input device to action relationship. Another way to look at the tables is to look at an example. The input device model 215

is used to describe a device that did not meet one or more of the elimination items. The numbers represent the device to action rating. The number is obtained by multiplying the task table weights to the concatenated device tables. The absolute value of the number is not as significant as the relationship of a number to that of another device. The higher the number in the table the less likely the input device in question is the optimal device for that particular action. The lower the number the more likely that input device is the optimal input device for that particular action.

\section{CONCLUSION}

The resultant table located in the AFIT archives shows the grade of the input devices for each action. Table 6 shows the optimal devices which was extracted from the resultant table. The first and fifth actions have the same input device as the optimal device which is the Ballpoint from MicroSoft. The reason for this is because the device is cheap, somewhat reliable, and has a button. Because the numbers were so close, the second most optimal input device was also highlighted which was the Logitech SpaceControlMouse. Actions two and three also have the same input devices as the optimal. PQ Control's 215 and 220 are the best because they are the cheapest device with a relatively high dexterity rating. The last two actions show the most dexterous input devices for two DOF and three DOF situations. It should be pointed out that dexterity can be a subjective rating. A user may like an input device better then another device and operate that device much better then another. With the open architecture that the Air Force's system will have, a user can pick his or her best device. The system would then handle calibrating that device into the architecture. If another user wants a different input

manufactured by $\mathrm{P} Q \mathrm{Q}$ Controls has the numbers extracted from the device tables and table 4 and listed in Table 5.

The table above is an example of computing the device to task relationship. The number at the lower right corner matches the number for action two (column 4 of the resultant table) and the P Q Controls model 215. If the device tables and task analysis table (Table 4) were used as matrices, then the solution of all devices and all actions are simply a matrix multiplication problem.

The resultant table provides the device to task relationship. The table consists of numbers or an $\mathrm{E}$. The $\mathrm{E}$ device, then again the system should handle calibrating the different input device into the architecture.

Since there is more then one device for an action, it is recommended that each device that is optimal for an action be purchased and interfaced into the Air Force's generic telerobotics architecture. The actions are dividable within the task so the combination of the input devices will not be confusing nor difficult to understand. The user can use PQ Control's model 215 to move the manipulator's platform into position, then use the Schilling six DOF controller to 
perform the manipulator control. Using more then one input device to handle the task gives the user flexibility in controlling the entire task. The user can control the platform with one hand while controlling the manipulator with the other. There are also other combinations that can be used to enhance the overall system performance.

\section{REFERENCES}

[1] NASA Jet Propulsion Lab (JPL) Pasadena CA. A Generic Telerobotics Architecture for C-5 Industrial Processes, Prepared for Air Force Materiel Command (AFMC) Robotics and Automation Center of Escellence (RACE). San Antonio Air Logistics Center, Kelly AFB. TX 78241.

[2] H. Asada and J. K. Salisbury. Telerobotics and Applications, 1991.

[3] A. Bejczy, "Teleoperatoin: The Language of the Hand". IEEE Intemational Workshop on Robot and Human Communication, pp. 32-43, IEEE Press, 1992.

[4] Thomas B. Sheridan, Telerobotics, Automation, and Human Supervisory Control. The MIT Press. Cambridge. MA. 1992.
Thomas E Deeter, Captain, USAF

Tom Deeter started his engineering career at Wright State University where he received his bachelors in Electrical Engineering in December 1989. He then worked for the Air Force as a radar analyst for 3 years. He received his masters from the Air Force Institute of Technology in December of 1994. He his currently working at the Robotics and Automation Center of Excellence in San Antonio Texas.

Dean L Schneider, Ph.D., Major, USAF

Dean Schneider is a member of the Air Force Institute of Technology Robotics and Automation Applications Group with research interests in telerobotic control and robotic reliability. He earned a Ph.D. in mechanical engineering from the University of Texas at Austin and an MSEE from the Air Force Institute of Technology. Dr Schneider has served as an ICBM maintenance officer and as a depot engineer in charge of reliability programs at the San Antonio Air Logistics Center. Dr Schneider is a member of the IEEE, Eta Kappa Nu, and the Air Force Association.

Table 6 Device to Action Relationships

\begin{tabular}{|c|c|c|c|c|c|c|c|}
\hline Company Name & Model & 1 & 2 & 3 & 5 & $6 \mathrm{~A}$ & $6 B$ \\
\hline \multirow[t]{6}{*}{ Cyber-tech, Inc. } & $8900 / 500$ & 368 & $E$ & $E$ & 368 & $E$ & Wly \\
\hline & $8600 / 500$ & 368 & $\mathrm{E}$ & $\mathrm{E}$ & 368 & $\mathrm{E}$ & 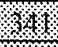 \\
\hline & $1800 / 500$ & 468 & $E$ & $E$ & 468 & $\mathrm{E}$ & W \\
\hline & $1200 / 500$ & 468 & $E$ & $E$ & 468 & $\mathrm{E}$ & W \\
\hline & $\begin{array}{l}\text { BRUT- } \\
88 / 500\end{array}$ & 368 & $E$ & $\mathrm{E}$ & 368 & $\mathrm{E}$ & \% \\
\hline & $300-\mathrm{H} / 500$ & 368 & $E$ & $E$ & 368 & $\mathrm{E}$ & 3/x \\
\hline Logitech & $\begin{array}{c}\text { Space } \\
\text { Control } \\
\text { Mouse }\end{array}$ & w. & 466 & 466 & $1 \%$ & 685 & 685 \\
\hline Microsoft Corp & BallPoint & 181 & $E$ & $E$ & $1 \%$ & $\mathrm{E}$ & 783 \\
\hline \multirow[t]{2}{*}{ PQ Controls Inc } & 215 & 207 & $\sqrt{3 / 3}$ & (3) & 207 & 364 & 369 \\
\hline & 220 & 207 & $\frac{1}{3}$ & m. & 207 & 364 & 369 \\
\hline $\begin{array}{l}\text { Schilling } \\
\text { Development Inc. }\end{array}$ & TITAN & 1011 & 1109 & 1109 & 1011 & $\%$ & $\mathrm{E}$ \\
\hline
\end{tabular}

